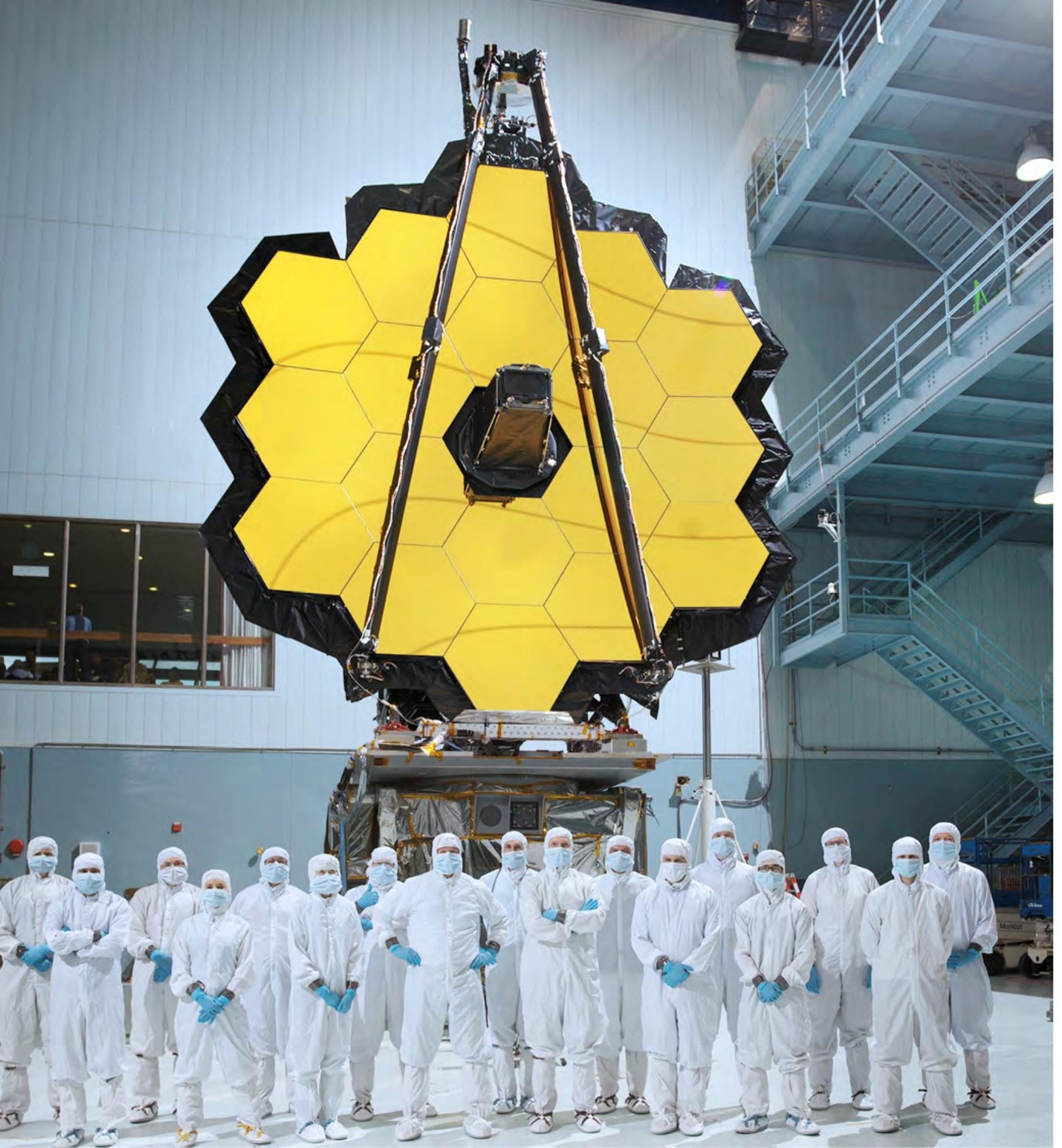

JWST Optical Telescope Element Center of Curvature Test Presented by Ritva Keski-Kuha NASA/GSFC 


\section{Test Team}

- B. Saif, R. Keski-Kuha, L. Feinberg, GSFC

- D. Chaney, K. Smith, L. Carey, Ball Aerospace

- M. Bluth, J. Bluth, A. Abeel, SGT

- P. Greenfield, K. Van Gorkom, K. Brooks, W. Hack, STScl

- S. Chaung, Orbital/ATK

- T. Sanders, T. Martinez, Vantage Systems Inc.

- S. Tournois, Sigma

- J. Berrier, Intelletechs

- M. Barker, C. Bowser, Genesis

- S. Smith, MSFC

- Vladimir Kradinov, SAO 


\section{JWST Overview}

- James Webb Space Telescope (JWST) is a large infrared observing space telescope which will launch in 2020.

- The observatory is comprised of 4 major subsystems

- Optical Telescope Element (OTE)

- Integrated Science Instrument Module (ISIM)

- Sunshield

- Spacecraft Bus

- The OTE and ISIM are integrated together at Goddard Space Flight Center to form the OTIS assembly

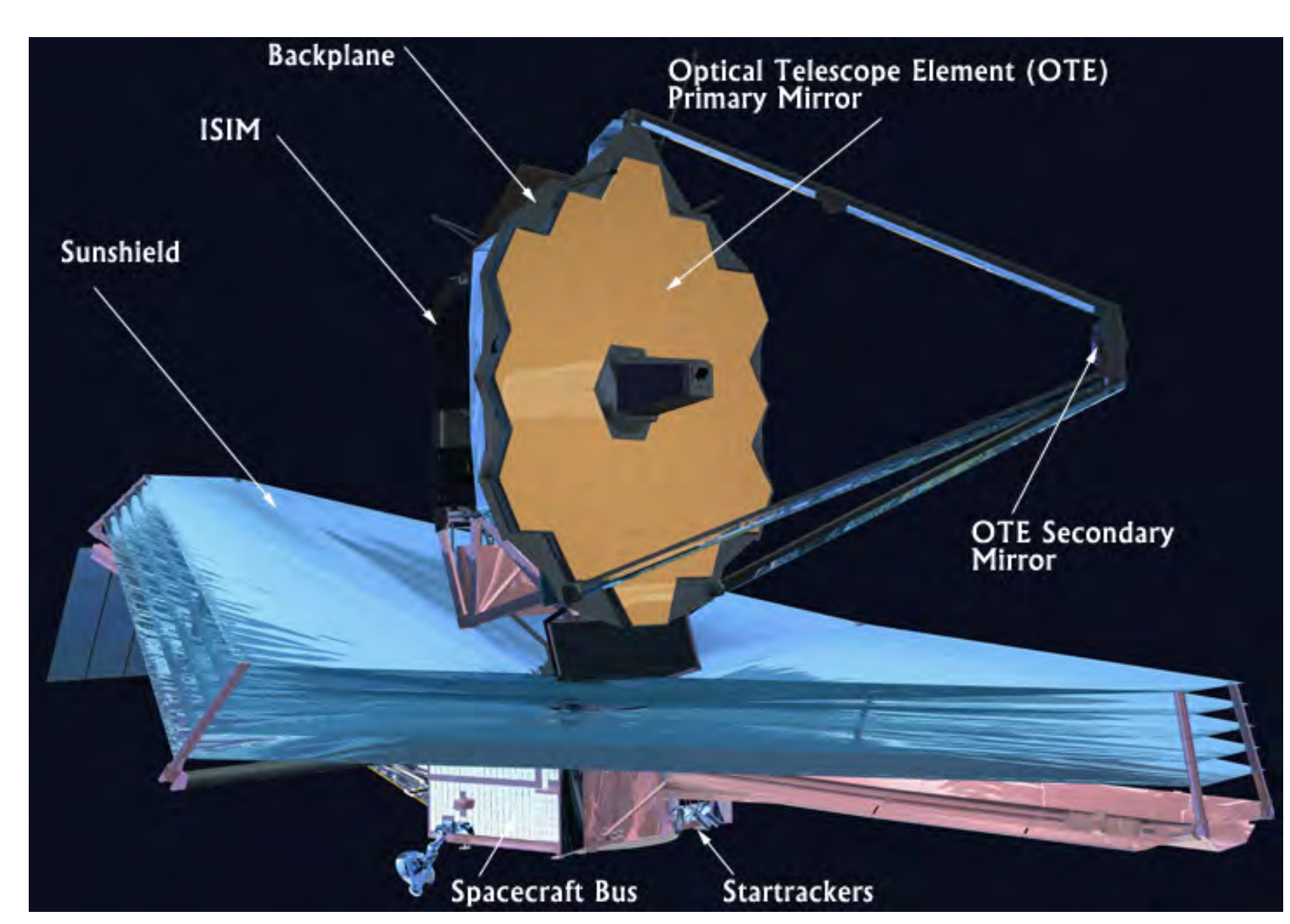




\section{OTIS Integration \& Test Summary}

- The OTIS assembly was integrated at GSFC and then went through a series of vibration and acoustic tests to verify its launch worthiness.

- To verify that no detrimental changes occurred to the OTIS assembly from this environmental testing an optical Center of Curvature $(\mathrm{CoC})$ test was conducted

- Help us in understanding potential anomalies identified during the OTIS cryo vac tests and will be helpful for future telescope design

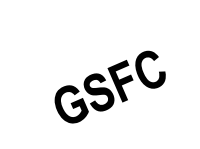

$$
\text { JSC }
$$

Northup
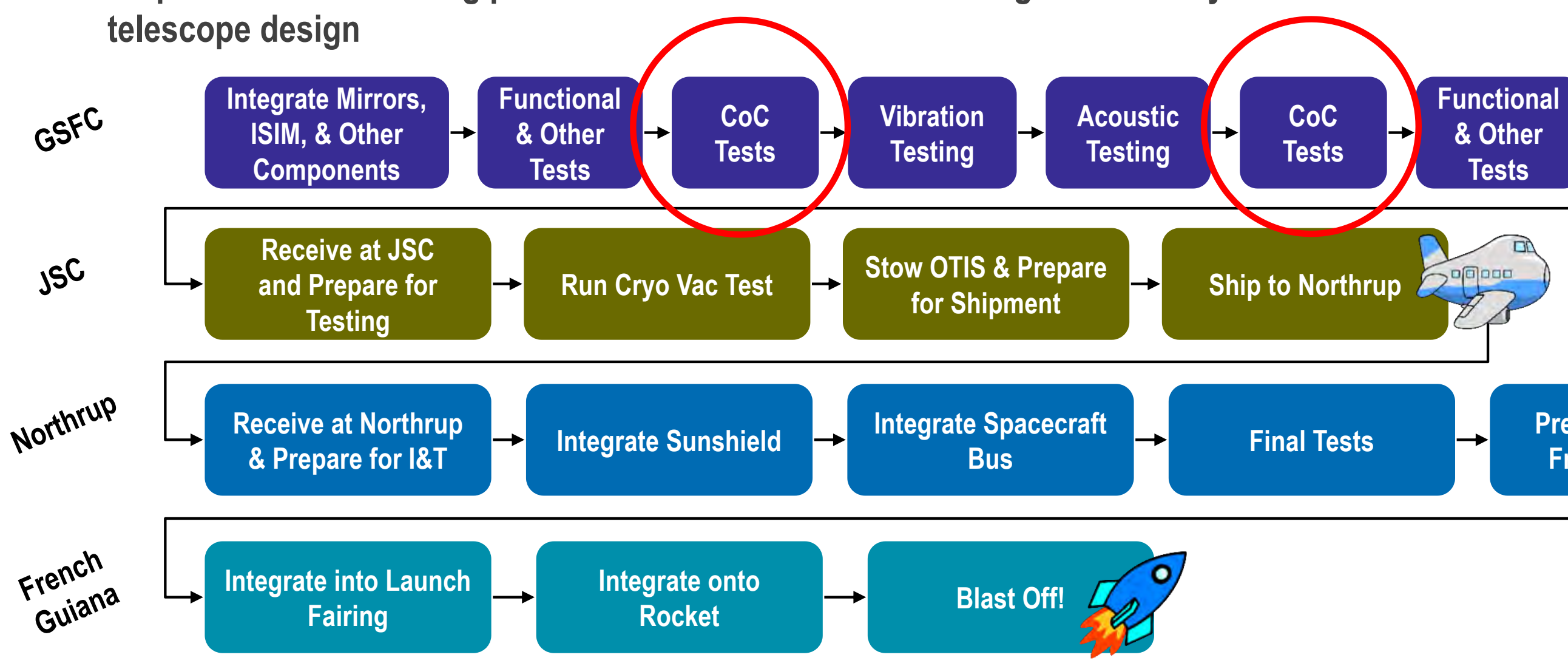

Fairing

Rocket 


\section{CoC Test Overview}

\section{- $\mathrm{CoC}$ (Center of Curvature) test is a standard type of optical test used to measure the}

surface figure of mirrors using an interferometer.

- For the SSDIF CoC test we expanded on this basic center of curvature test methodology by using a high speed interferometer manufactured by 4D Technologies capable of taking more than 5,900 surface figure measurements every second.

- We measured one mirror at a time (i.e. no phasing of mirrors)

- We performed two types of tests

- Static Test: measures the surface figure of the PM segments (one at a time) and looks for changes in the figure of the mirror. (resolution $=1520 \times 1520$ )

- Dynamic Test: takes up to 59,000 surface figure measurements over a 10 second period while applying a vibrational input force (stinger) to the OTIS backplane and then calculates changes to the phase and gain transfer functions. This is like having 40,000 one-axis accelerometers on each mirror segment. (resolution = 240x240) 


\section{Basic Test Layout}

Test Wavefront Matches Shape of "Perfect" Mirror, therefore all rays are normal to the mirror surface and retrace their path back into interferometer.

Test beam is also used to align CGH to interferometer

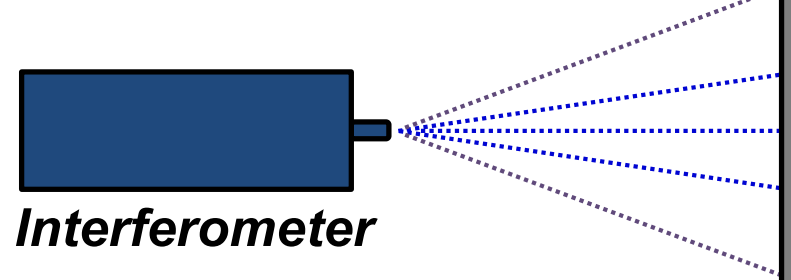

Measures difference in shape of mirror vs test wavefront

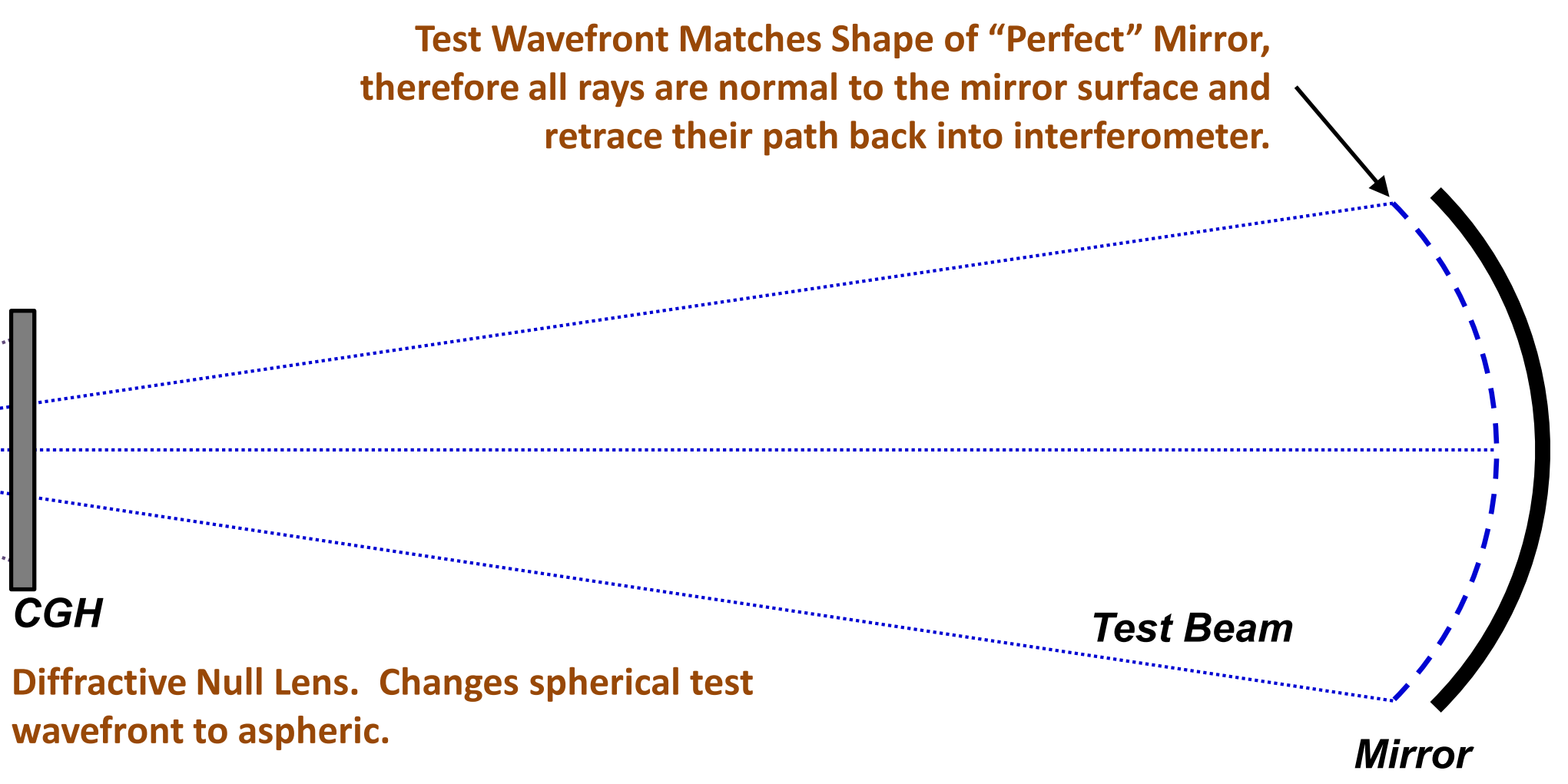




\section{Picture of Actual Test Layout}

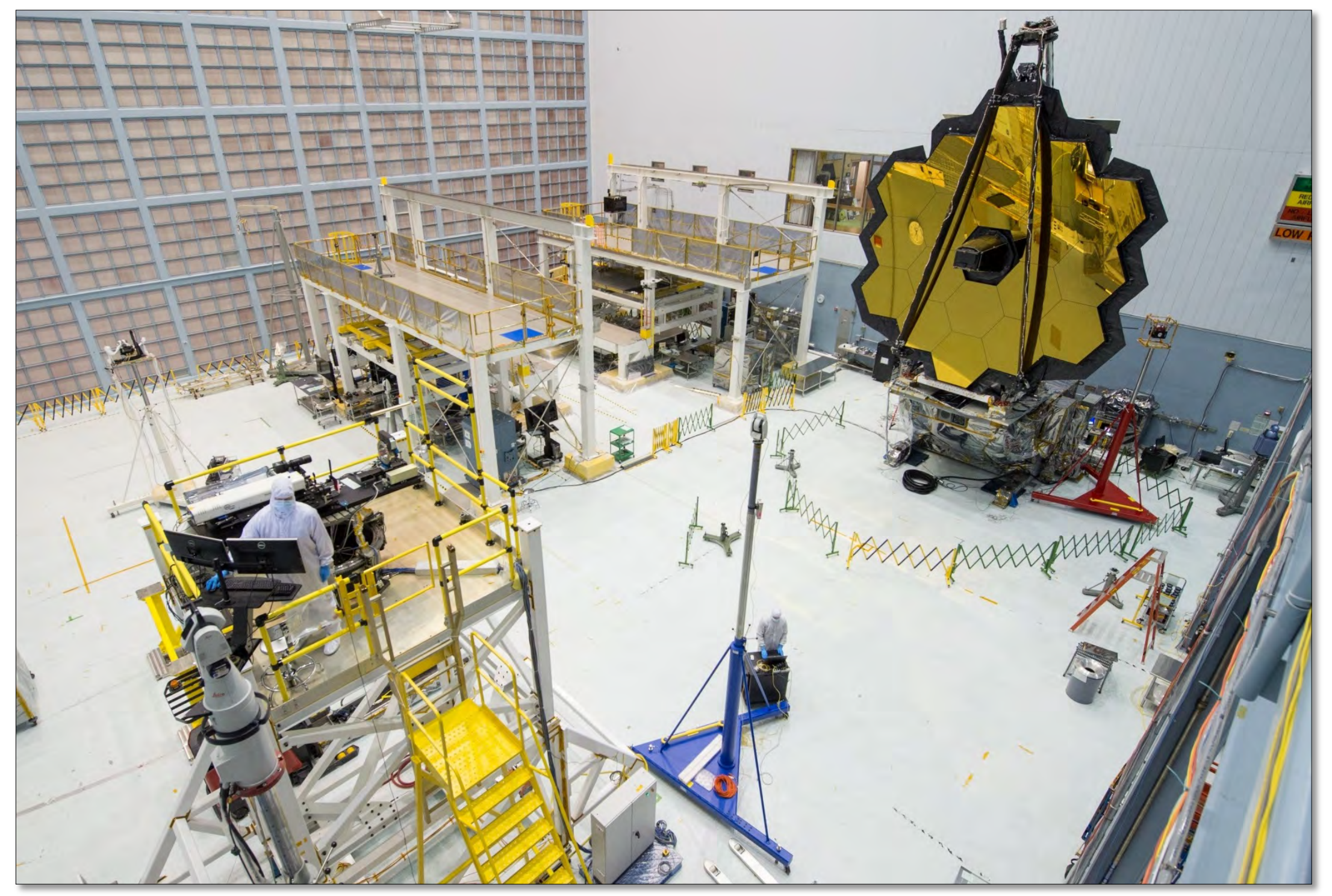




\section{Metrology Setup}
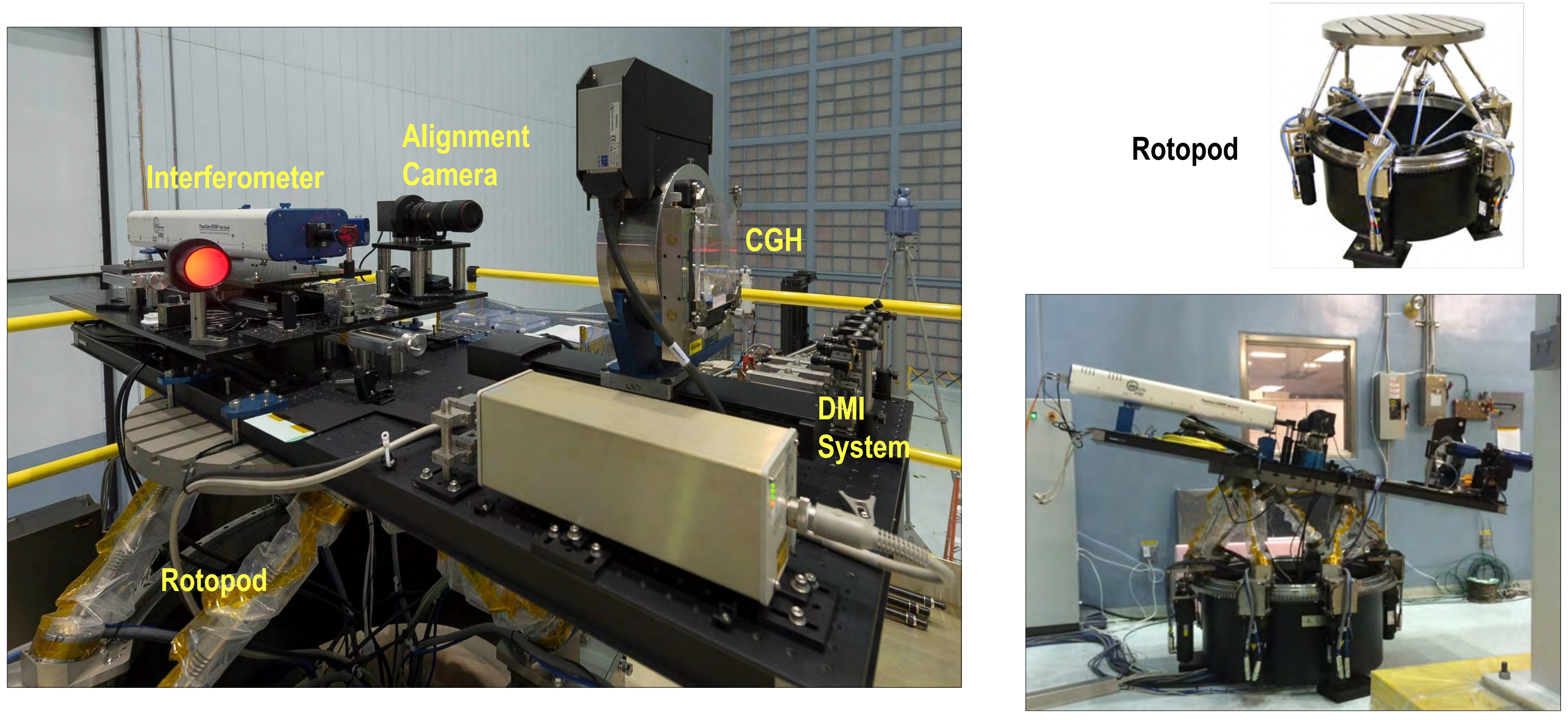


\section{Alignment Camera System}

- An newly developed alignment method was used to align the mirror under test to the CGH in 6 DoF.

- Did not require adding fiducials to the sides of mirror segments (they are only 6-7 $\mathrm{mm}$ apart)

- Used for matching alignment condition during pre and post environmental $\mathrm{CoC}$ testing

- A camera system took two images

- First image is of illuminated mirror

- Second image is of 4 Laser spots projected from CGH onto mirror surface

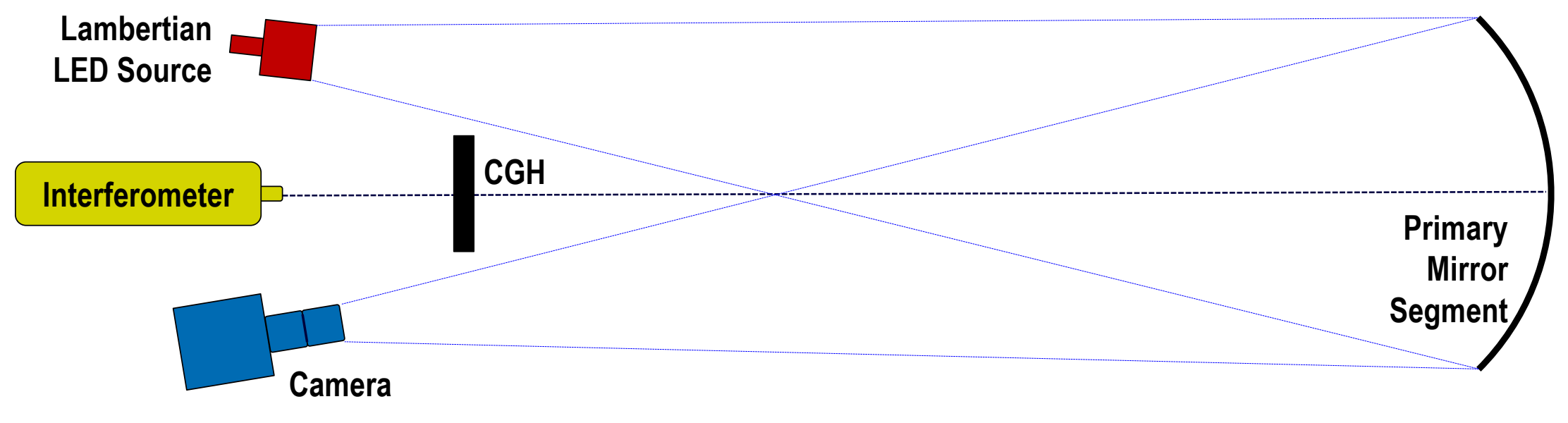




\section{Alignment Camera Images}

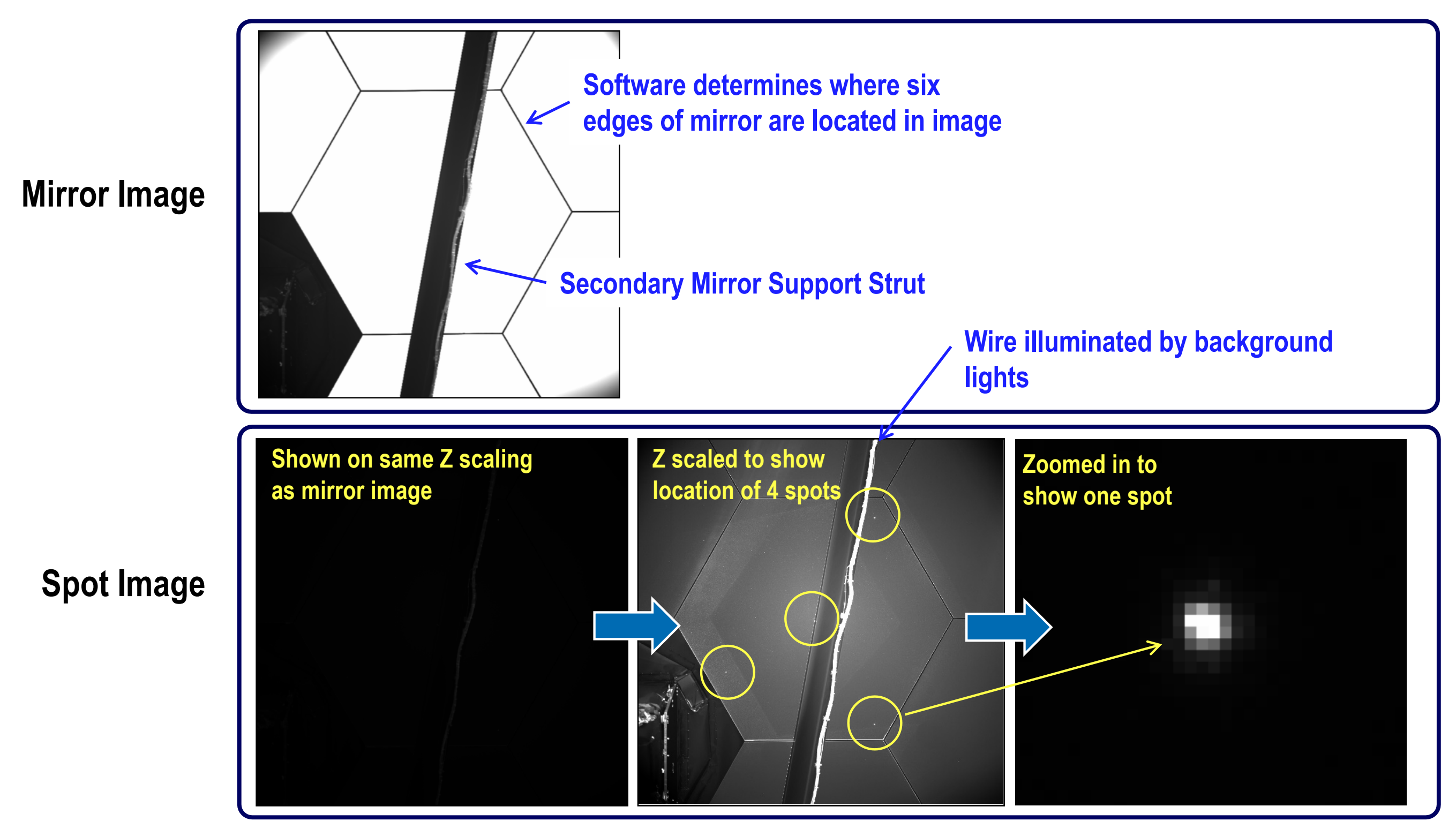




\section{Dynamic Testing Shaker/Stinger}

- A vibration shaker is used to apply a low level force into the composite backplane structure.

- Stinger (connection from shaker to mirror) is a graphite/epoxy rod to prevent sagging over the long distance

- A force gauge is attached between the stinger and backplane to capture input forces
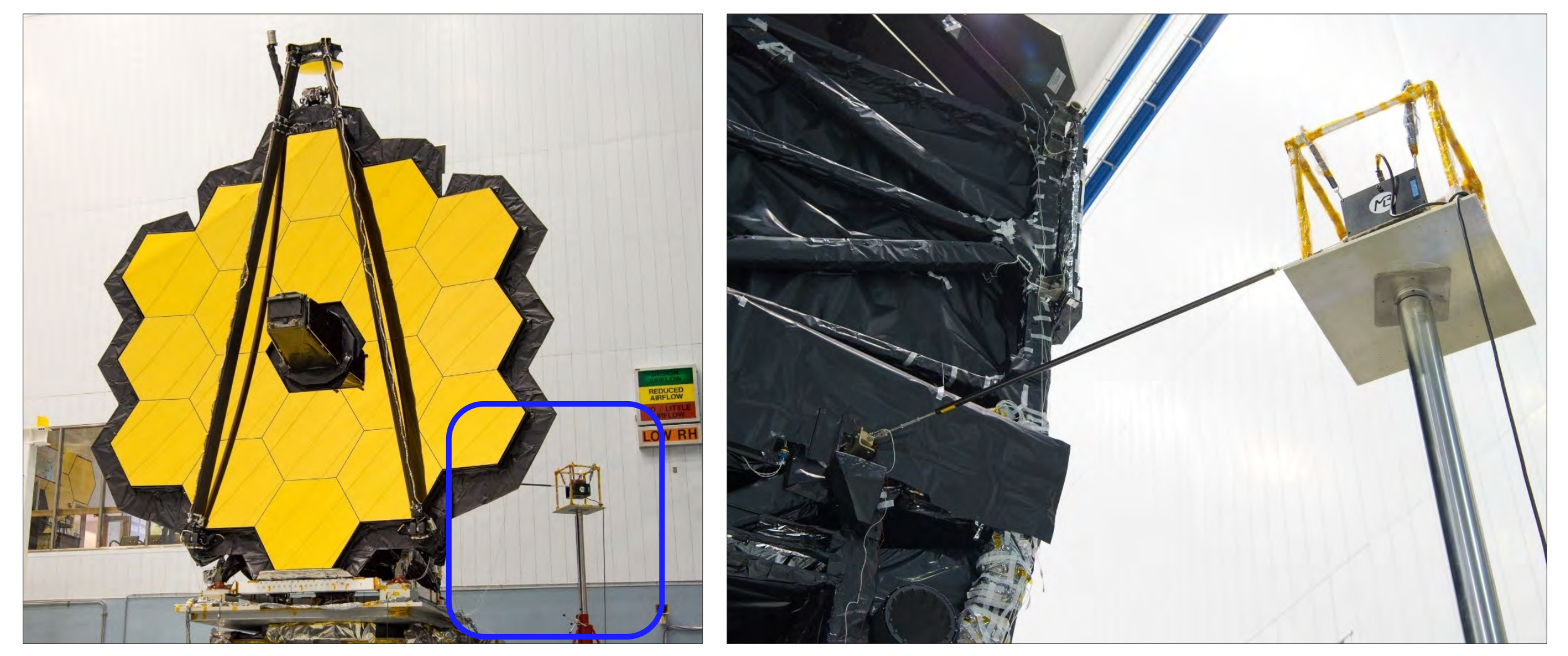


\section{STATIC MEASUREMENT RESULTS}




\section{Mirror Nomenclature \& Coordinate System}
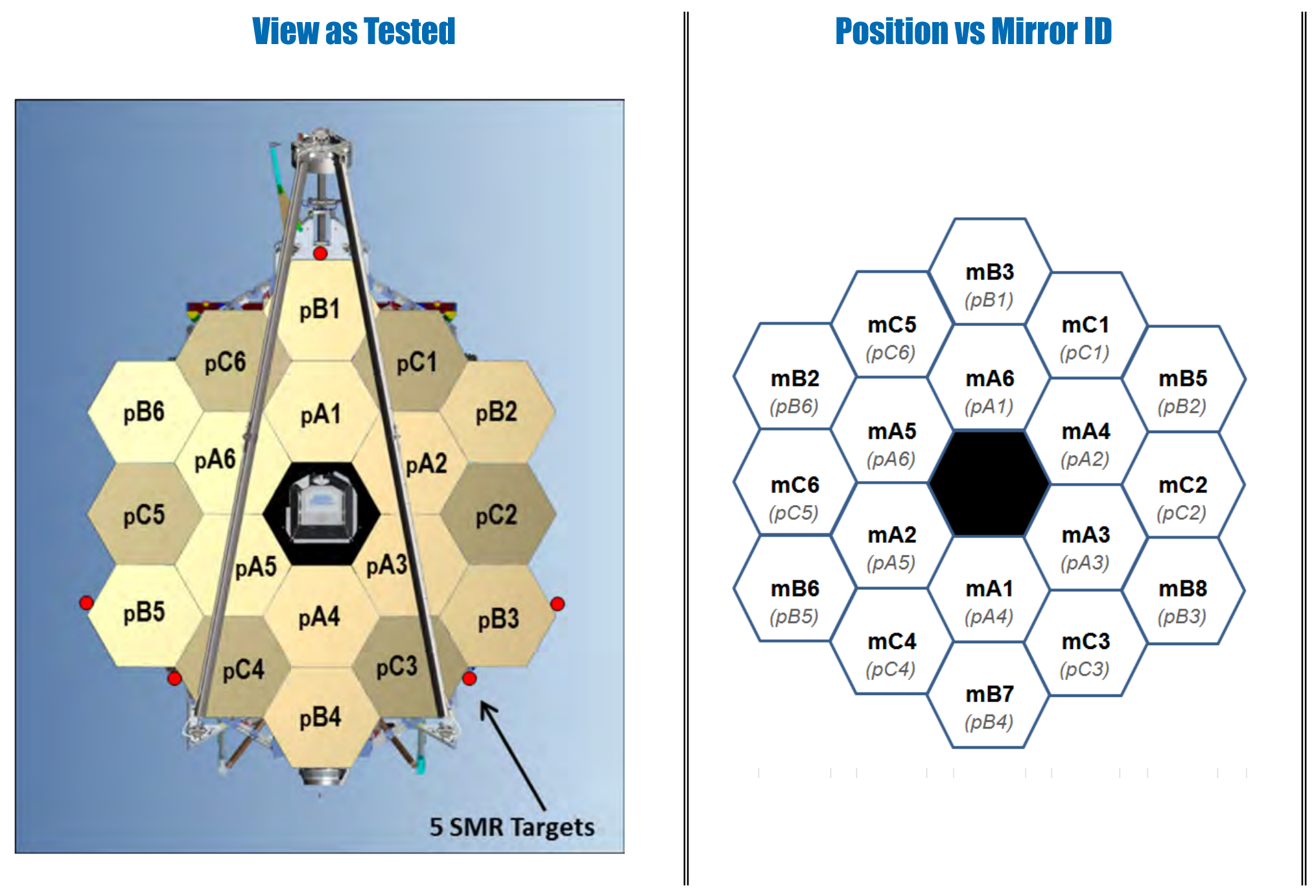

Mirror Coordinate System
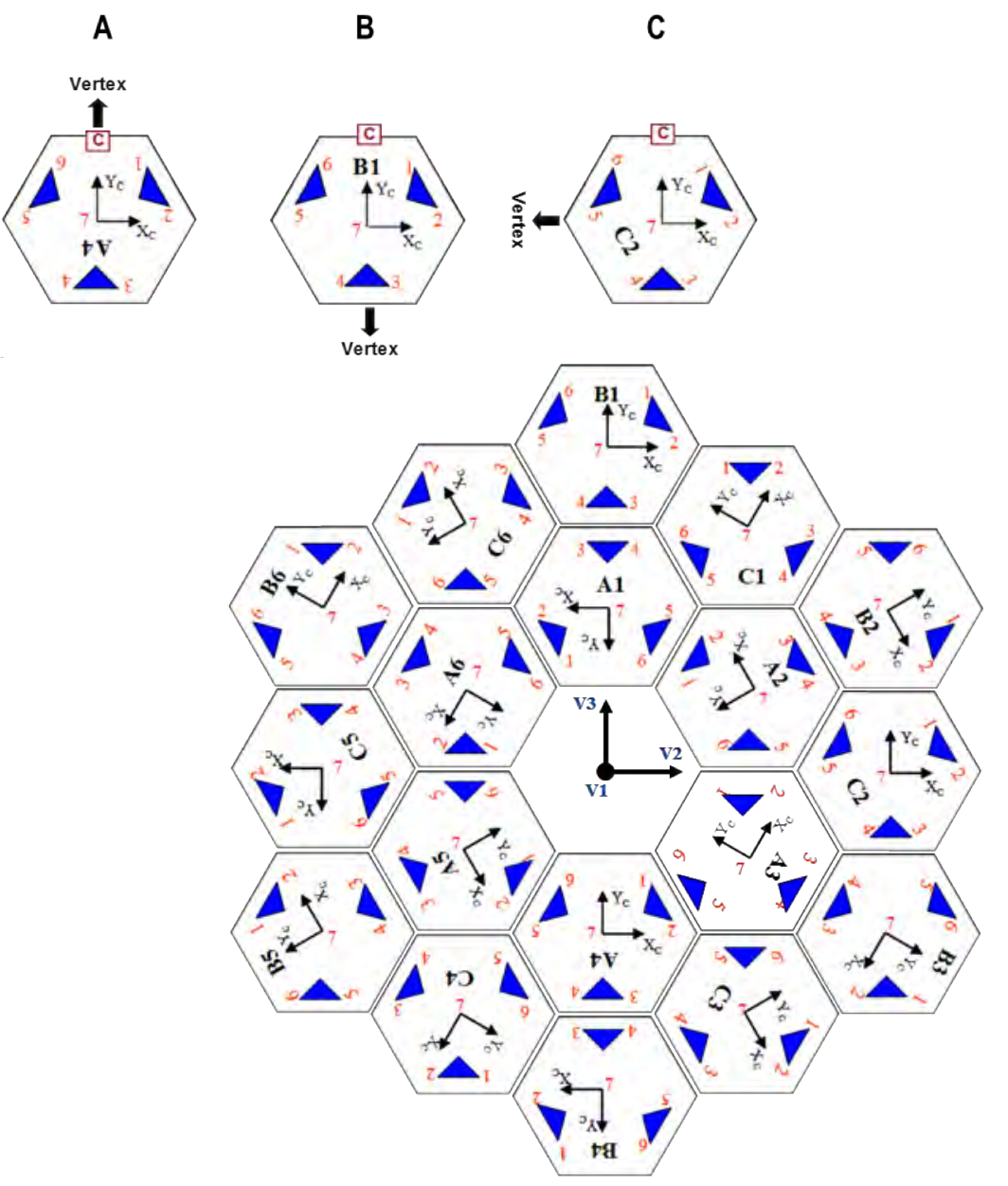


\section{Composite Image of Surface Figure Changes}

All Measured Changes were under our metrology uncertainty

\begin{tabular}{|c|c|c|c|}
\hline \multicolumn{4}{|c|}{ Metrology Uncertain } \\
\hline & A & B & C \\
\hline Figure & 12 & 12 & 12 \\
\hline
\end{tabular}

* Measured 4 times

** Measured 2 times

${ }^{* * *}$ Modified Alignment Method

Removed from Data

Piston, Tilt, Power, Astigmatism

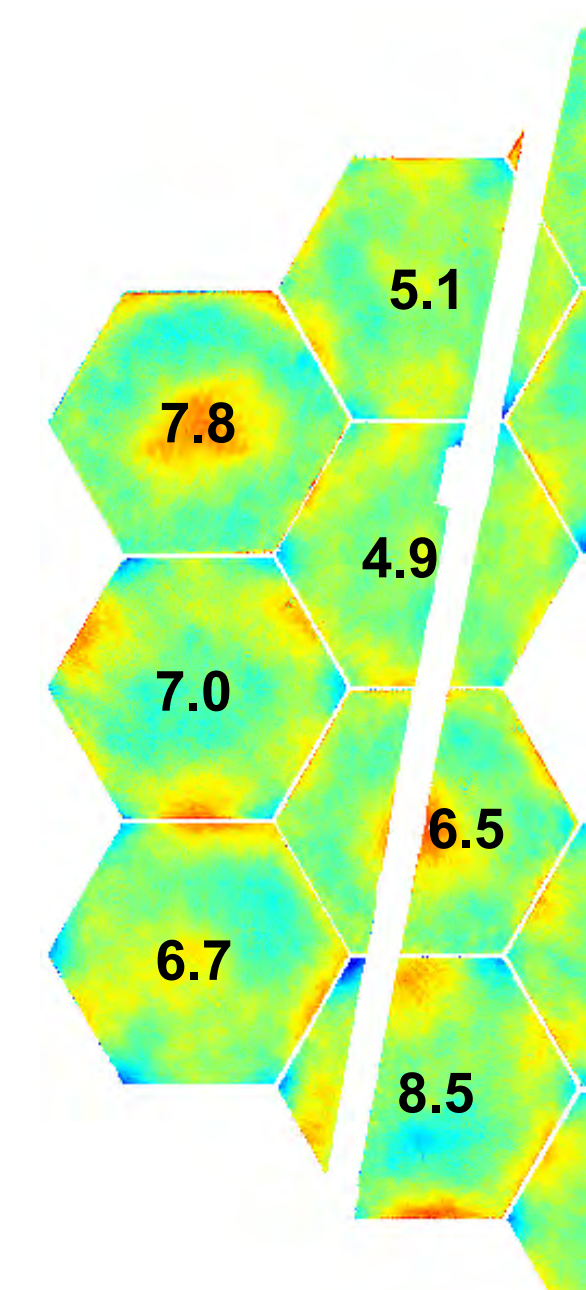

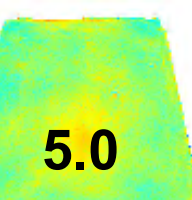

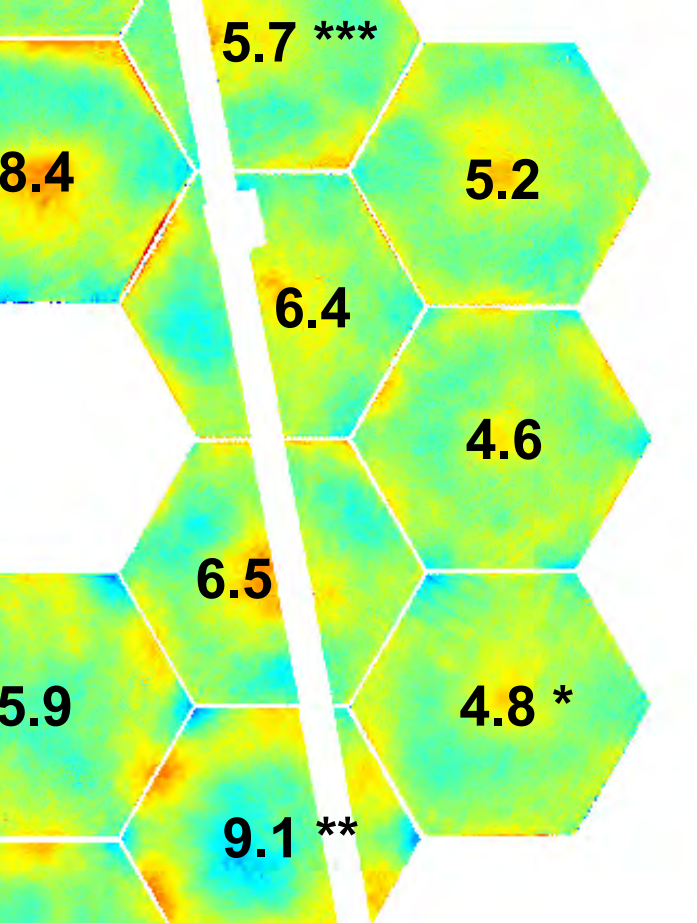

5.4 **
RMS:

\section{$6.5 \mathrm{~nm}$}

PV:

$106.8 \mathrm{~nm}$

Units $=\mathrm{nm}$ rms

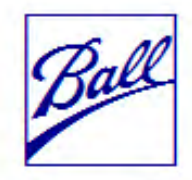

52.0

$\mathrm{nm}$

$-54.8$ 


\section{Composite Image of Astigmatism Changes}

All Measured Changes were under our metrology uncertainty

\begin{tabular}{|llll}
\multicolumn{4}{|c|}{ Metrology Uncertainty } \\
& A & B & C \\
\hline Astig & 26 & 53 & 45 \\
\hline
\end{tabular}

* Measured 4 times

** Measured 2 times

${ }^{* * *}$ Modified Alignment Method

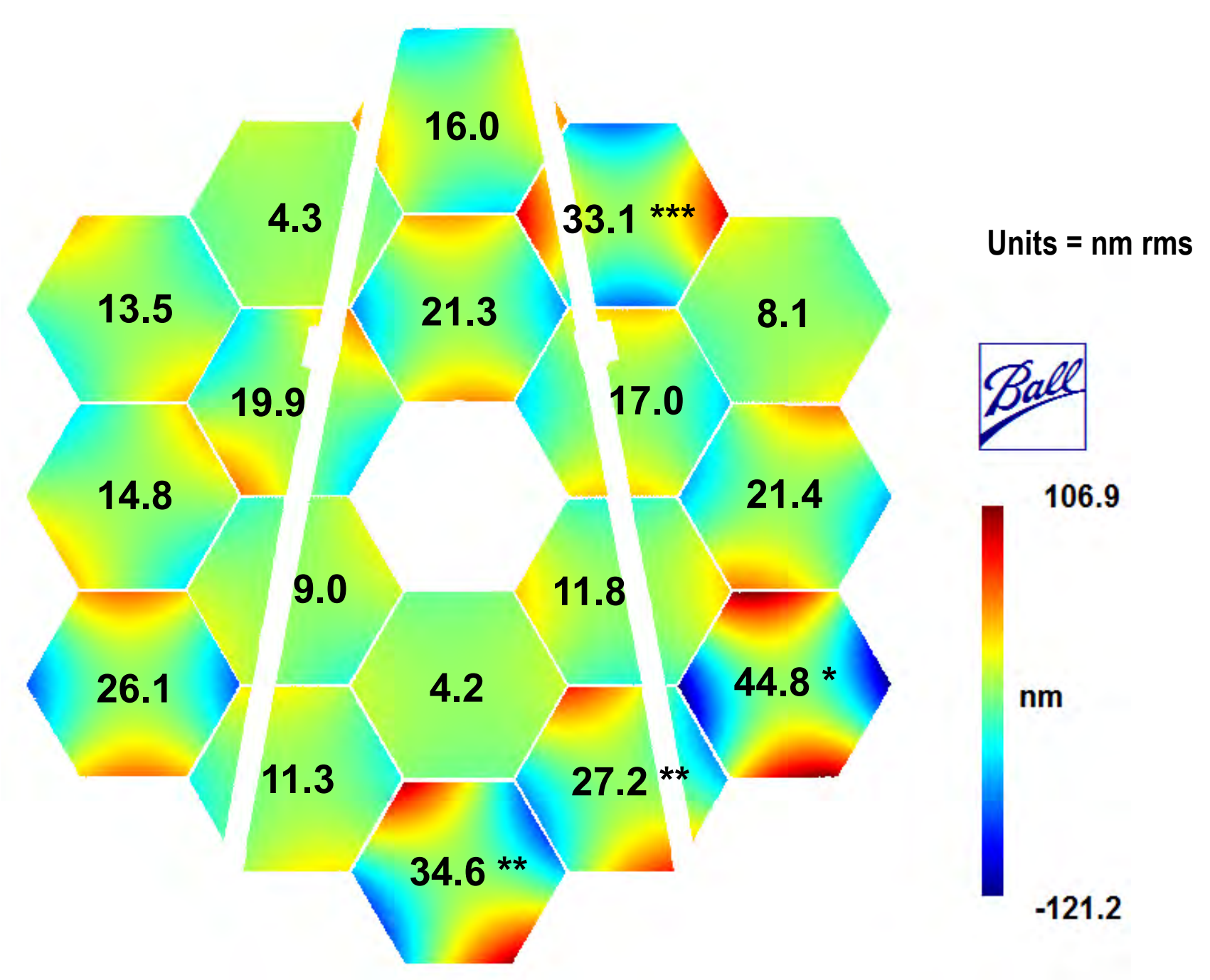




\section{Additional Static Results Analyses}

\section{- pC1 results looked a little high for astigmatism (33nm rms vs 45 uncertainty)}

- This was probably due to an alternate method used to align mirror. The Secondary Mirror Support Struts interfered with our alignment features (projected CGH spots) and therefore we applied an approach of rotating the $\mathrm{CGH} 180^{\circ}$ for alignment and then rotating back for the actual measurement. This added to astigmatism measurement error.

- Lower right 3 mirrors (pB3, pC3, pB4) showed higher astigmatism levels than other segments

- Two potential error sources were looked at.

- Heating from ISIM electronics unit was shown, through testing, not to be the source of the error.

- Alignment camera system shown to be a "potential" source of error. Additional testing showed that additional uncertainties due to room lighting, illumination source power, camera \& software settings needed to be accounted for in metrology uncertainty budget. 


\section{DYMAMIC MEASUREMENT RESULTS}




\section{Dynamic Measurement Block Diagram}

- The main goal of the dynamics CoC test is to acquire diagnostic survey data of the OTIS vibrational characteristics at low input levels

- Background with no input stimulus,

- Sine Sweep over $25-50 \mathrm{~Hz}$ or $10-50 \mathrm{~Hz}$

- Random input.

- The CoC dynamics test uses low level of forcing functions on order of $10 \mathrm{~N}$ or less. This force was a dynamic load applied to the OTIS

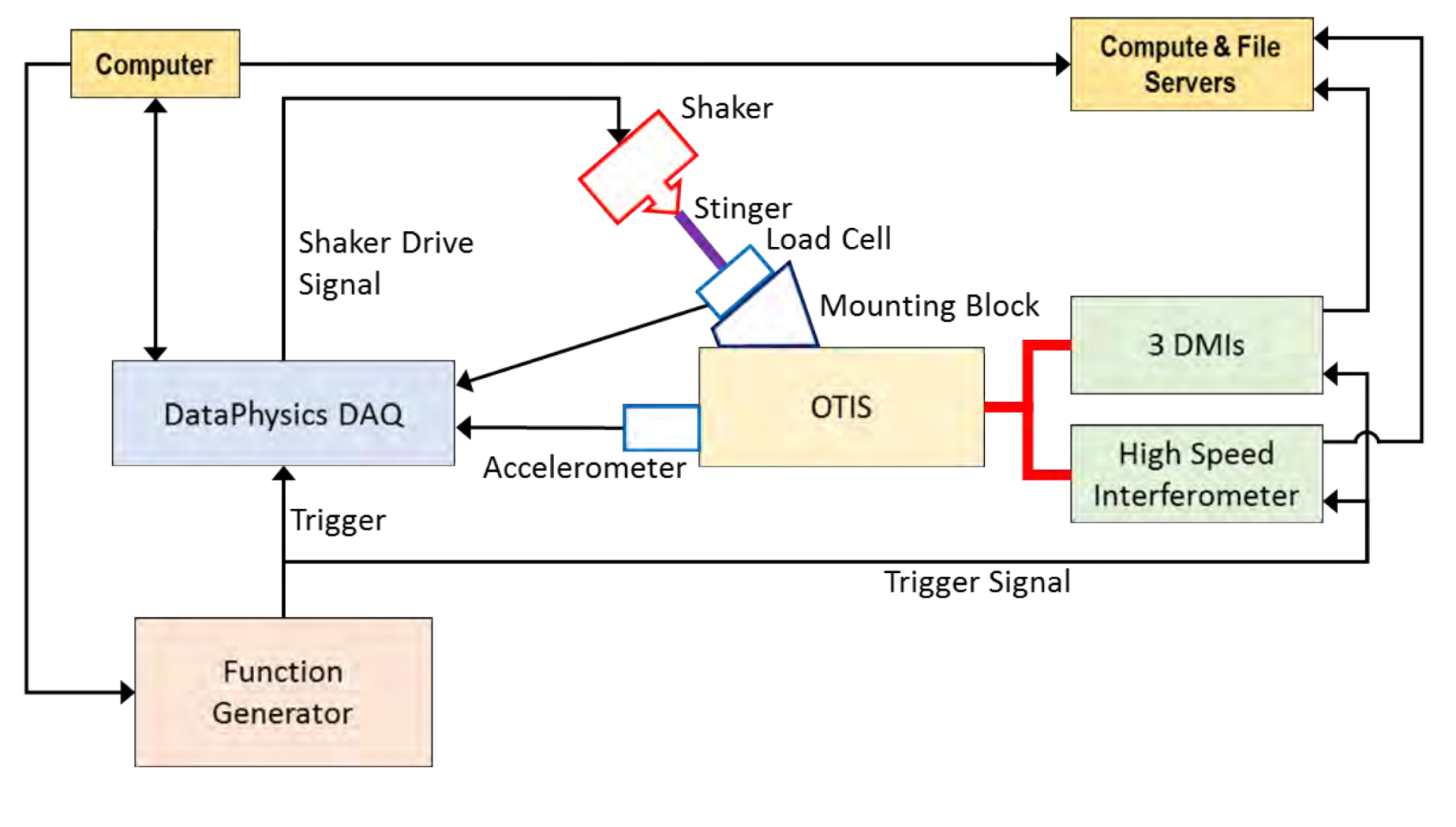
composite structure while the HSI observed a PMSA 


\section{Dynamic Data Processing Flow}

\section{Processing Flow Chart}

(for a single dataset)

$4 \times 59000$ instantaneous hase-shifted interferograms

(10 seconds at $5.9 \mathrm{kHz})$

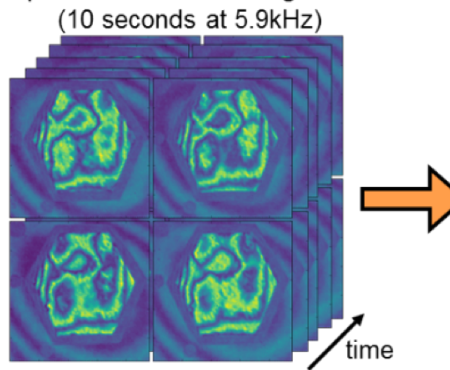

wrapped phase cube

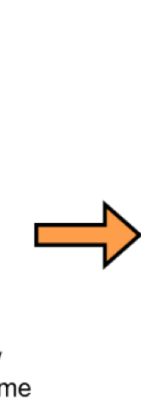

time-series displacement cube

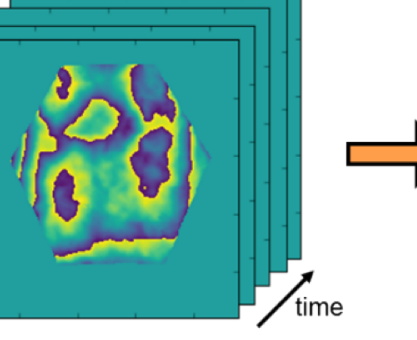

undistort from CGH frame to MMR

Zernike decomposition of each frame

to produce time-series coefficients
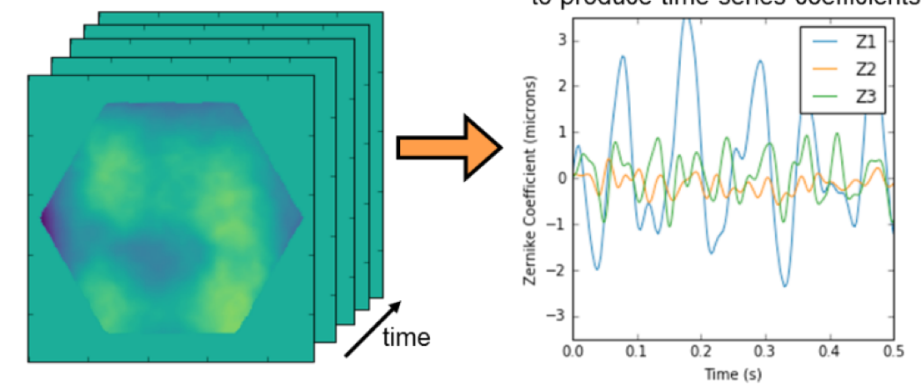

\section{Processing Flow Chart}

(combining datasets)

Compute the transfer function $\mathrm{H}(\mathrm{v})$ to allow comparison mong datasets with different stimulus levels and phase

$H(\nu)=\frac{R(\nu)}{F(\nu)}$ Zernike Coefficient FFT

$H(\nu)=\overline{F(\nu)} \leftarrow$ stimulus FFT

TF Gain: $g(\nu)=|H(\nu)|$

TF Phase: $\phi(\nu)=\arctan \left(\frac{\operatorname{Im}[H(\nu)]}{\operatorname{Re}[H(\nu)]}\right)$

Organize into groups ( $\sim 10$ datasets) with common stimulus and average
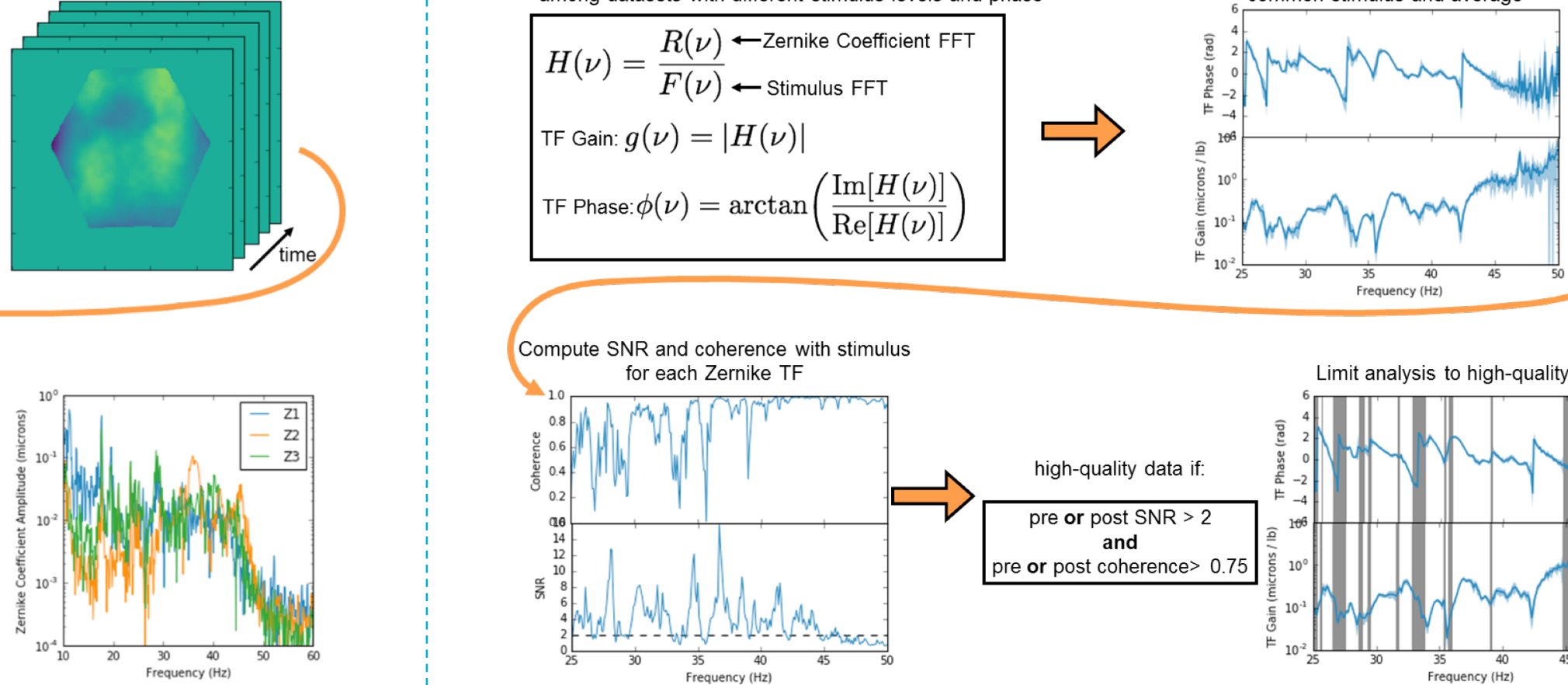


\section{Example of Primary Mirror Response}

Rigid Body (Z1-Z3) at $43.0 \mathrm{~Hz}$

Transfer Function Gain

Preshake

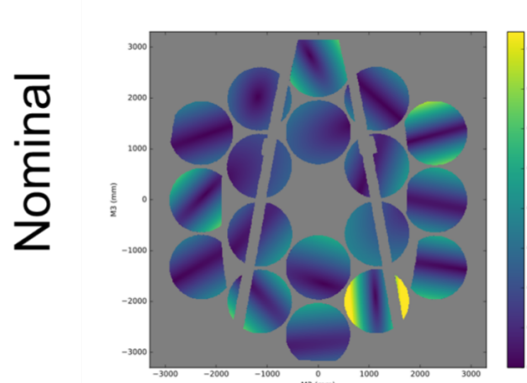

Postshake
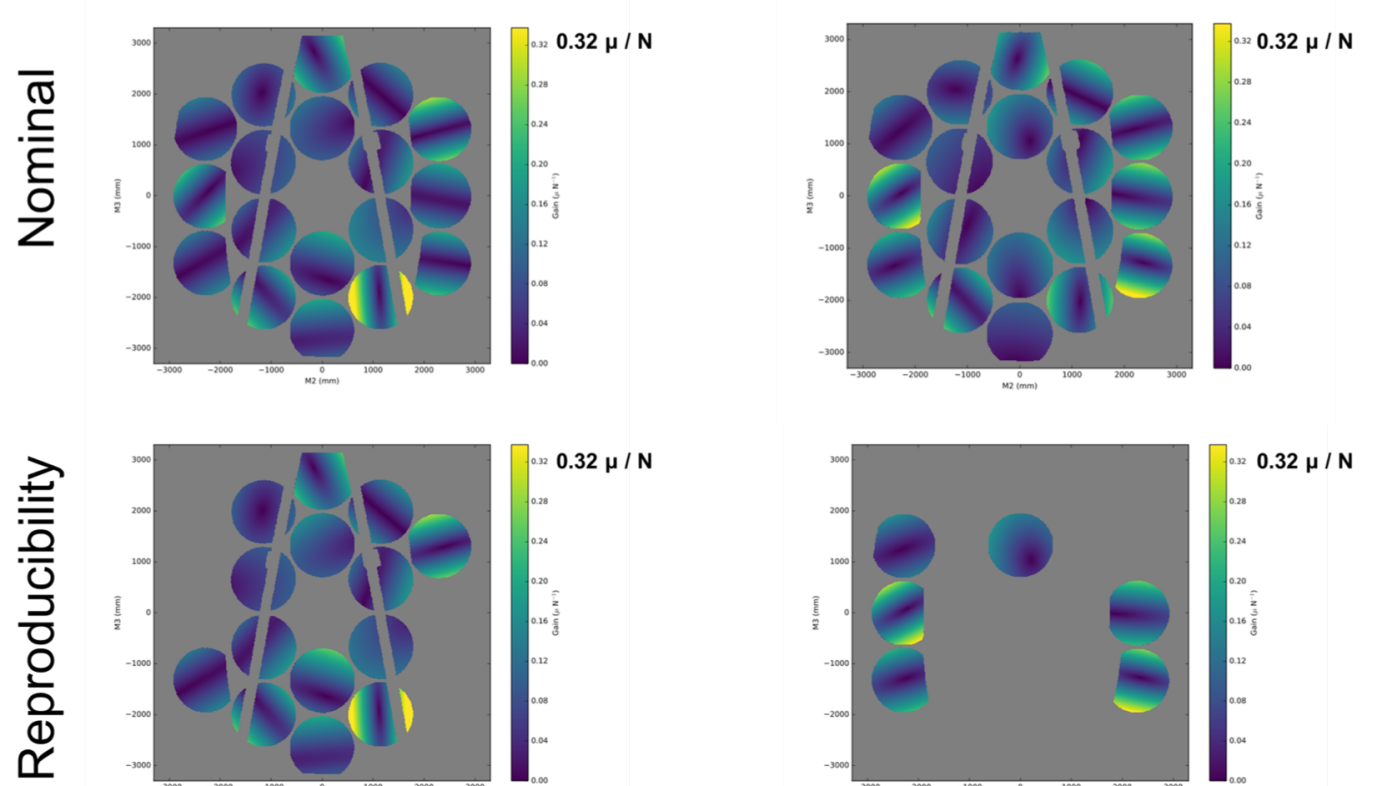
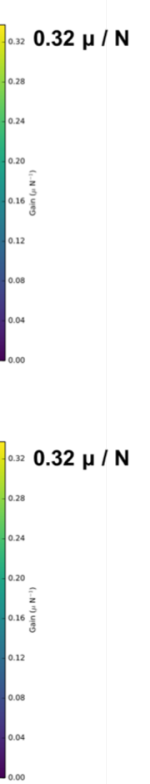

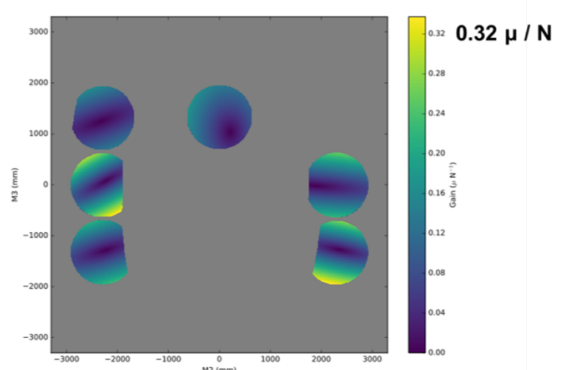

Rigid Body (Z1-Z3) at $43.0 \mathrm{~Hz}$ Transfer Function Phase

Preshake
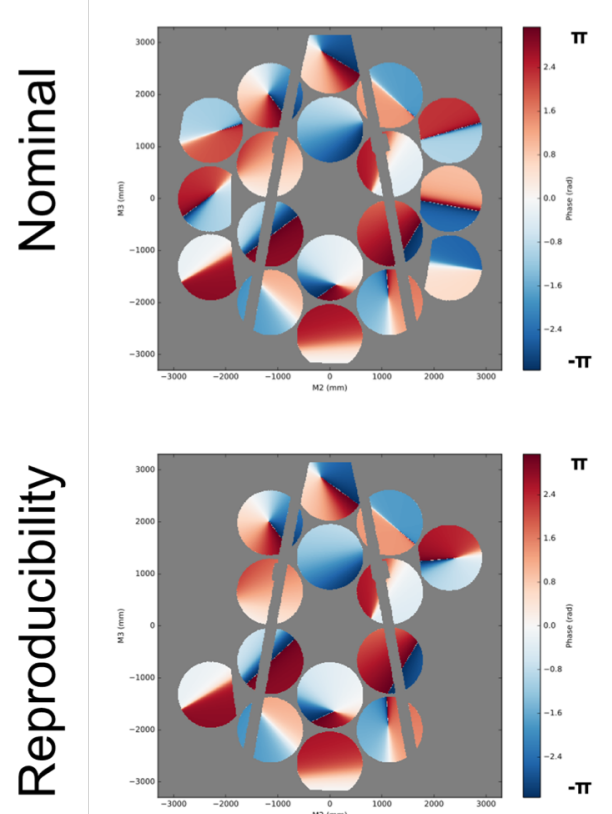

Postshake
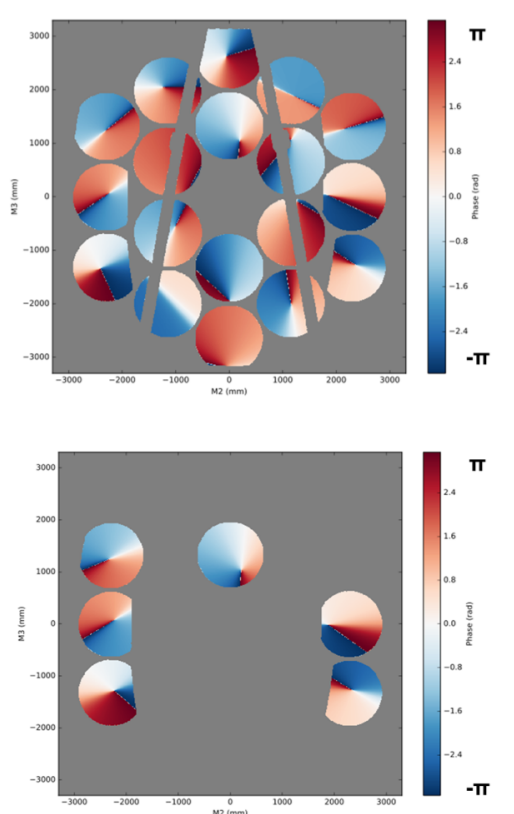


\section{Example of Primary Mirror Response}

Astigmatism (Z5-Z6) at $43.0 \mathrm{~Hz}$

Transfer Function Gain

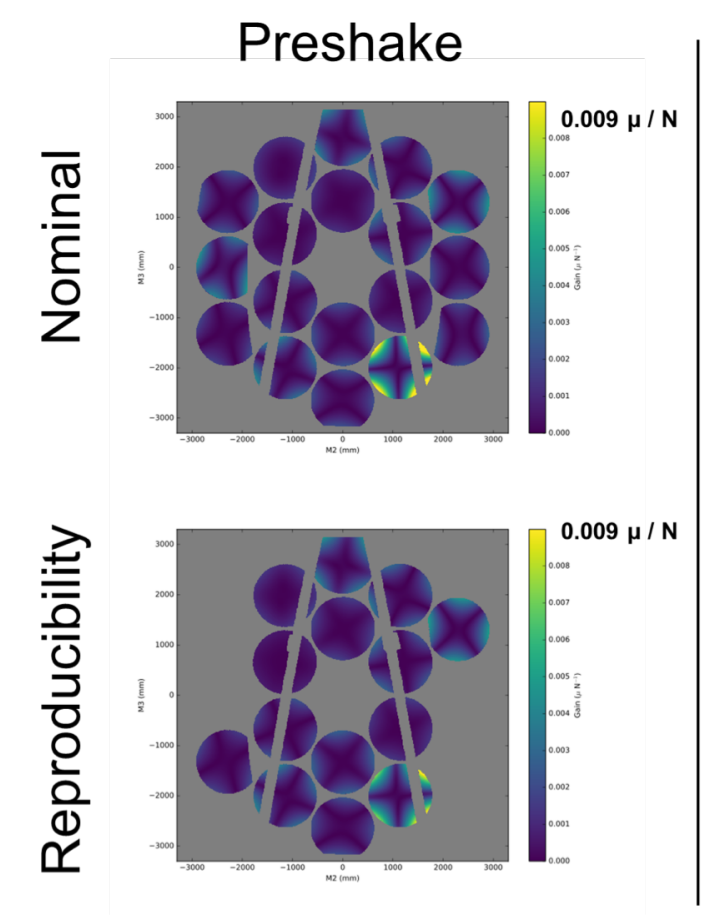

Astigmatism (Z5-Z6) at $43.0 \mathrm{~Hz}$ Transfer Function Phase
Preshake
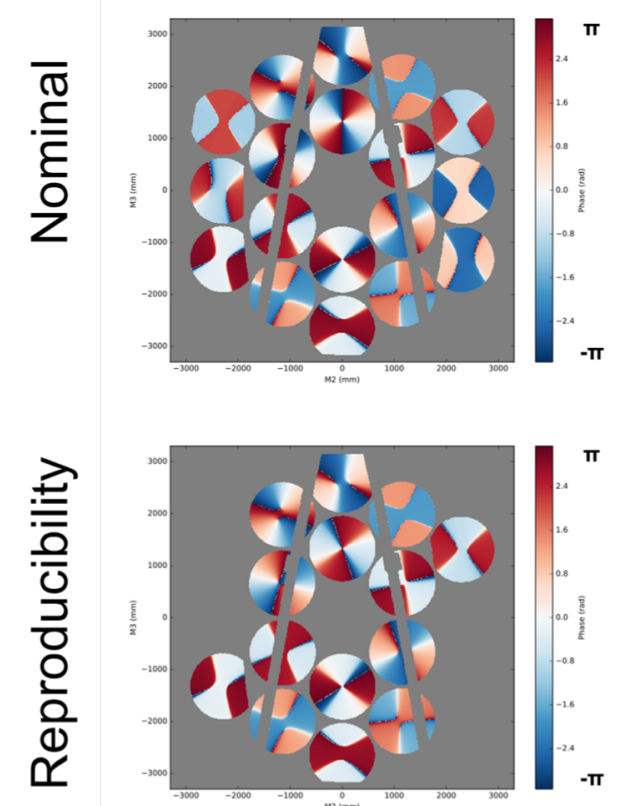

Postshake
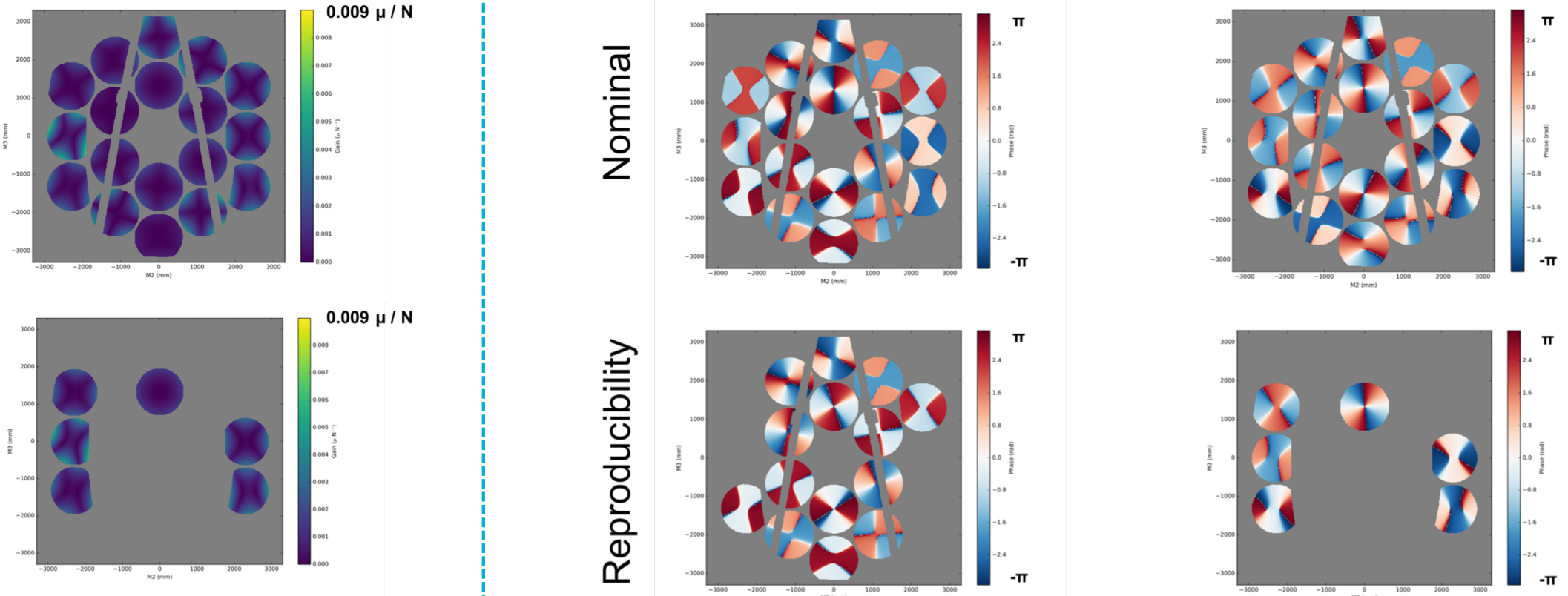


\section{Conclusion}

- The Center of Curvature test was successful in verifying that no unacceptable changes occurred to the JWST telescope assembly as a result of vibro-acoustic testing.

- The static portion of the test provided excellent results given the enormity of the test.

- A newly developed alignment camera system worked well.

- The dynamics portion of the CoC test successfully measured the opto-mechnical modes of the telescope in low amplitude stimulation to nanometer precision.

- Informed about the health of the OTIS before shipping it to Johnson Space Center (JSC) for optical testing at cryogenic temperature. 